\title{
VIEWS OF CHILDREN, YOUNG PEOPLE AND FAMILIES: THE 15 STEPS CHALLENGE
}

\section{Open access}

\section{The first steps patients make into wards tell them a great deal about what to expect. Sian Thomas and Dave Clarke describe} how to involve them in making improvements and redesigns

Correspondence sian.thomas14@wales.nhs.uk

Sian Thomas is nurse consultant children and young people, Aneurin Bevan University Health Board, Royal Gwent Hospital, Newport

Dave Clarke is professional head of children and young people's nursing, Cardiff University and a member of the editorial advisory board of Nursing Children and Young People

Date of submission June 52015

\section{Date of acceptance}

July 82015

\section{Peer review}

This article has been subject to open peer review and has been checked using antiplagiarism software

\section{Author guidelines} journals.rcni.com/r/ ncyp-author-guidelines

\begin{abstract}
There is a growing emphasis on involving service users in improving service delivery, accelerated by the inquiry into care failings at Mid Staffordshire NHS Foundation Trust. The 15 Steps Challenge was originally developed by the NHS Institute for Innovation and Improvement as a tool to help healthcare professionals, service users and others to work together to improve the patient experience. The 15 Steps Challenge involves a ward 'walkaround' to see the ward through the eyes of a child or young person and a parent or carer. This article explores the emphasis that is placed on user involvement in the context of children's nursing, and shares the experience of planning and evaluating the introduction of the 15 Steps Challenge in an acute children's ward in a busy district general hospital.
\end{abstract}

\section{Keywords}

child health, 15 steps challenge, paediatric nursing, patient care, service quality, service user views

PATIENT AND service-user involvement has been talked about in healthcare organisations for many years, but awareness of poor standards of care at Mid-Staffordshire NHS Foundation Trust and the subsequent recommendations made by the Francis inquiry (Francis 2013) accelerated the quest for greater involvement of patients in service evaluation and re-design.
Capturing the views and experiences of service users is a key element of how health organisations can measure their performance, and demonstrate learning and service improvement. NHS Wales and the Welsh Government have identified this as a key priority. All seven local health boards in Wales must now demonstrate and provide assurance on how they have listened to and will meet the needs of service users to improve health outcomes (NHS Wales 2013).

Health organisations need to capture and demonstrate service-delivery improvement for their whole client population. It is, therefore, imperative that a range of methodologies is considered that capture the voice of children, young people and their families in a range of settings. Involving service users can happen at many levels and this article includes a discussion of an initiative where professionals have engaged and worked with families to review and redesign services.

\section{Involvement}

Seeking the views of children and young people is of fundamental importance because it is a basic human right enshrined in the United Nations Convention on the Rights of the Child (United Nations 1989). Article 12 sets out the right of all children and young people to be able to express an opinion and to have that opinion taken into account in any matter that affects them. The convention encourages adults to listen to the opinions of children and involve them in decision making, 


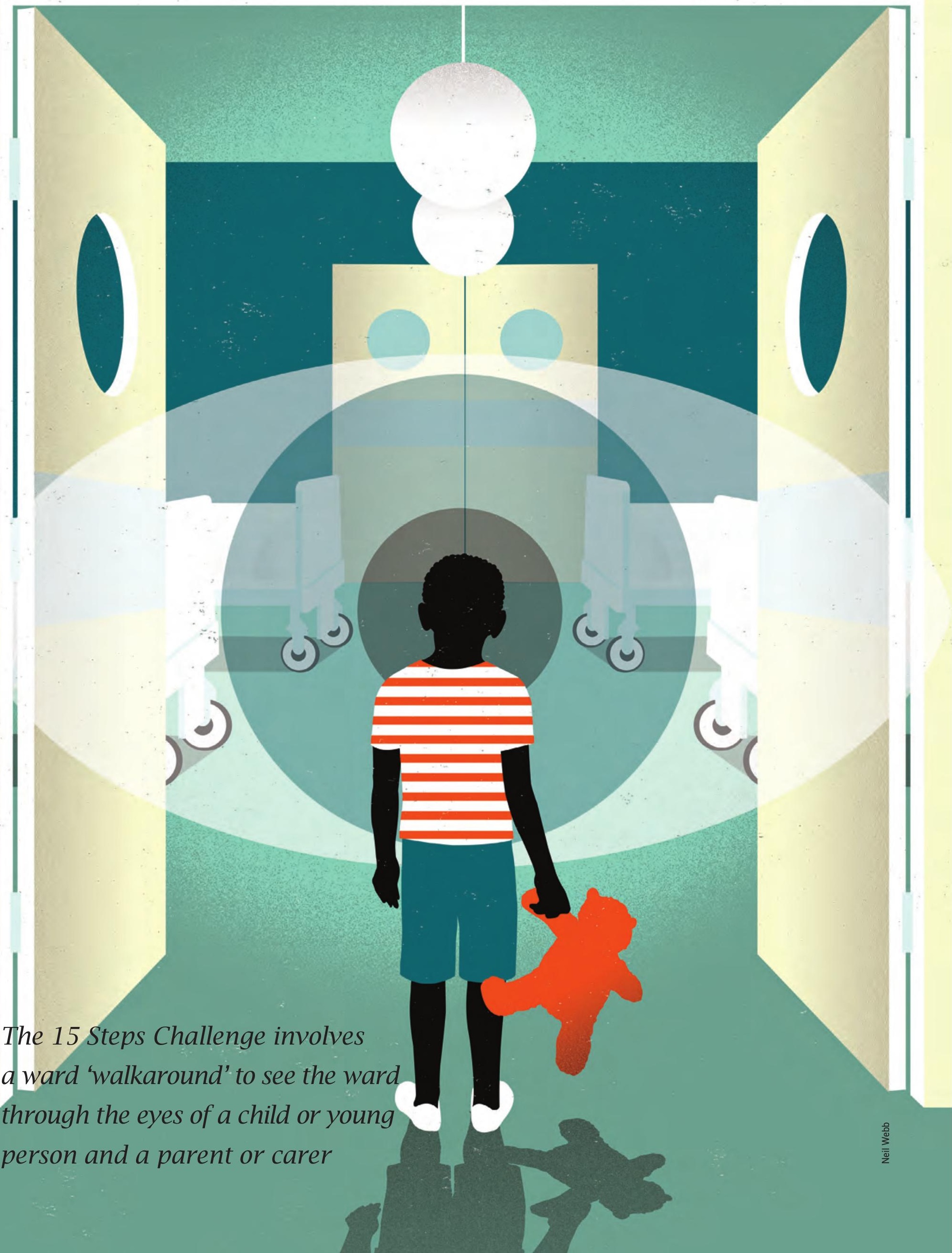


recognising that the level of a child's participation in decisions must be appropriate to the

child's level of maturity.

This is supported in Wales through the introduction of the Rights of Children and Young Persons (Wales) Measure (Welsh Government 2011), which places a duty on Welsh ministers to consider children's rights. This ministerial responsibility encourages the development of a mainstream children's rights culture within the work of the Welsh Government and, ultimately, in wider Welsh society.

In recent years, there has been a significant commitment across health, social care, education and the voluntary sector to involve young people more actively in having a voice and a choice in matters that affect them. As a result, a range of resources has been developed that enables or supports the participation of children and young people in line with the Children and Young People's National Participation Standards for Wales.

These standards have been established to help improve the process for children and young people to be involved in participation and decision making. Children and young people need to be offered various methods to participate that are appropriate to their age.

The NHS Institute for Innovation and Improvement (2013) has developed a proactive approach to seeking the views of children and young people about inpatient services and this article explores how this approach has been implemented in practice.

\section{The 15 Steps Challenge}

A mother of a child in hospital commented to healthcare staff on the importance of first impressions, as service users quickly know whether they have confidence and trust in the care service they are using. Her comment, 'I can tell what kind of care my daughter is going to get within 15 steps of walking on to a ward', inspired the creation of the 15 Steps Challenge (NHS Institute for Innovation and Improvement 2013).

The NHS Institute for Innovation and Improvement developed the 15 Steps Challenge,

\section{Box 1 The 15 Steps Challenge toolkits}

Acute services

Mental health inpatient care

Children and young people's inpatient services

Community services

Clinic and outpatient settings

(NHS Institute for Innovation and Improvement 2013) one of a suite of toolkits (Box 1), in partnership with service users, healthcare staff and board members to redesign and drive quality improvements as part of the Productive Care workstream for NHS England. This was part of the Quality, Innovation, Productivity and Prevention (QIPP) national workstream (NHS Institute for Innovation and Improvement 2013) which aimed to ensure that patients are cared for in the most productive environment, for example a ward, theatre or their own home. The NHS Institute for Innovation and Improvement closed on March 312013 and has been replaced by NHS Improving Quality.

The 15 Steps Challenge implemented at Aneurin Bevan University Health Board includes varying degrees of participation for children, young people and their families and, although the idea for the challenge was initiated by an adult, the tool was developed in partnership with young people. The 15 Steps Challenge helps healthcare professionals, service users and others to work together to identify improvements that will enhance the patient experience. The challenge involves a ward 'walk-around', seeing the ward through the eyes of a child or young person and parent/carer.

The 15 Steps Challenge toolkit consists of a poster, observation guide and feedback sheet that helps facilitate a service evaluation, clearly documenting all the items that children and families say matter to them during admission. Following an explanation of the process by the 15 Steps Challenge co-ordinator, the team is asked to consider what good quality care looks, sounds and feels like from a child's and a family's perspective using the observation guide and feedback sheet.

The guide and sheet concentrate on the four key themes that families have identified as important:

Is the ward welcoming?

Is it safe?

Will it care for me?

Is it well organised and calm?

Following the ward walk-around, the co-ordinator feeds back findings to the clinical area, focusing on good practice and areas for improvement. This process is then repeated on a regular basis, with the frequency largely depending on the outcome of the walkaround. The aim is to ensure that improvements are sustained and that there is progression.

\section{Implementation}

Recognising the potential value of the 15 Steps Challenge toolkit for children and young people's inpatient services, senior nursing staff 
adapted and piloted the toolkit within the Aneurin Bevan University Health Board.

One of the five children's areas was chosen for the pilot as it was the largest ward and provided care for children up to 18 years. Acknowledging that the average length of stay as an inpatient on the children's unit is generally one day, the team were unsure whether there would be a suitable patient available and prepared to participate in the walk-around. To address this, a young person was invited from a local youth forum. There is no specific health forum within the area so a request was made to the generalist youth forum for someone with no previous experience of hospitalisation to participate in the walkabout.

Feedback from all members of the challenge team was positive as they felt really involved and empowered by the process and welcomed the equal partnership that existed between team members. The ward staff felt it was beneficial to receive direct feedback as this helped them focus on the areas for improvement that really matter and make a difference to service users.

\section{What was learned}

During the ward walkaround, the 15 Steps Challenge team highlighted areas of good practice and areas that need improvement. These included:

visible engagement with staff and service users Friendly, open, helpful and approachable staff encourages service users to feel safe and involved.

\section{Environment}

Murals and soft colours produced a homely, positive feel but more consideration needed to be given to treatment areas, which appeared starkly different from the main ward area, potentially quite frightening for children.

\section{Quality of information}

It was felt that information was well presented and clear. The team liked the public health information leaflets that were available.

\section{Identifying staff}

The team liked the photo board and the friendly photos but suggested moving the board to a more visible area and using it to identify the staff on duty that day more clearly.

\section{Signage}

It was felt that this could be improved. It would be helpful for families to understand what rooms are for and which places they are allowed to access.

The 15 Steps Challenge co-ordinator fed back to the nursing team in a staff meeting and was received positively. Suggestions were made about how to address the ideas for improvement and how a 15 Steps noticeboard could be used to feed back to families.

\section{Conclusion}

As health professionals, nurses are in a prime position to support the engagement of children and families to influence service developments. This takes time but can be rewarding as it ensures their views about ways to improve and transform healthcare services are heard. Staff need to be innovative in their approach to meeting the needs and abilities of service users - one size does not fit all.

Involving service users is beneficial to them and to professionals. Children and families learn new skills and feel empowered, and as professionals nurses receive the assurance that services are being developed to meet the needs of families. Staff liked

\section{Points for practice}

Capturing feedback from services users can help healthcare organisations to measure their performance and demonstrate improvement

A growing number of resources, including the 15 Steps Challenge, are available to help give a voice to children and young people in matters that affect them

A simple observation guide and feedback sheet can be used to gather the views of children and young people and parents/carers on various aspects of healthcare

Improving and transforming health services requires staff to be innovative in their approach to meeting needs - one size does not fit all

Involving service users in improvement empowers them but also assures staff that families are benefiting from changes made to service delivery

\section{Find out more}

Resources that support the participation of children and young people are available at www.participationworkerswales.org.uk

the 15 Steps Challenge approach as it helped capture the service improvements made and it is hoped it can be adopted in other inpatient areas.

\section{References}

Francis R (2013) Report of the Mid Staffordshire NHS Foundation Trust Public Inquiry. Executive Summary. The Stationery Office, London.

NHS Institute for Innovation and Improvement (2013) The Fifteen Steps Challenge: Quality from a Patient's Perspective. tinyurl.com/8fyzlrb (Last accessed: September 14 2015.)

\section{Online archive}

For related information, visit our online archive and search using the keywords

Conflict of interest None declared 